\author{
Rosine Chandebois \\ Hubert Condamine
}

\section{Débat sur la notion de programme génétique de développement}

Existe-t-il

un programme

génétique de développement? Rosine Chandebois est toute prête à penser que non, que le développement de l'embryon est aussi peu programmé que l'est l'organisation sociale progressive d'une société humaine; l'acquisition de nouvelles structures dépendrait non pas d'une programmation génétique générale et souveraine, mais plutôt des interactions successives entre les éléments de l'organisme qui se construit. Hubert Condamine admet que le terme de "programme génétique " est probablement impropre, et devrait au moins être entendu comme étant d'ordre supérieur à $I$ : il s'agirait d'un programme de déroulement de plusieurs programmes élémentaires ... Il n'en reste pas moins que les exemples récents qu'apportent les études sur la drosophile d'un contrôle génétique précis de la morphogenèse ouvrent des perspectives jusqu'à présent inexplorées sur les mécanismes complexes du développement.

\section{Une notion aujourd'hui contestée}

Depuis quelques années, on commence à douter de la réalité d'un programme génétique, codé dans l'ADN, qui déterminerait les modalités du développement et entretiendrait les caractères d'organisation chez l'adulte. R. Lewin [I], en exergue d'un article très remarqué a écrit : "The more biologists learn about development the less it appears that organisms are assembled by neat, sequential processes». Le titre de cet article va au devant du scepticisme que suscitent des vues si contraires aux théories classiques : pourquoi le développement est-il si illogique? A coup sûr parce que nous lui avons fabriqué une logique qui satisfait la nôtre mais qui n'est pas la sienne. Les controverses sur cet hypothétique programme génétique sont le plus souvent argumentées par la biologie moléculaire $[2,3,4]$. Or, c'est l'embryologie qui donne les objections les plus intéressantes [5], en même temps que des solutions nouvelles [6].

La mise en route de l'organogenèse chez les amphibiens est des plus instructives. L'œuf en segmentation 
comporte deux "moitiés", l'une " ectodermique" (avec l'hémisphère animal), l'autre "endodermique " (les grosses cellules à vitellus). Séparées suffisamment tôt, ces deux moitiés dégénèrent l'une et l'autre sans s'être différenciées, bien qu'à ce stade n'importe quel noyau montre encore les propriétés de celui de l'œuf. Mais, dans le développement naturel, les cellules à vitellus déterminent, par une seule induction, les ébauches mésodermiques (chorde, muscles, rein, sang) aux dépens d'une partie de la moitié ectodermique (le reste, hors de portée de l'inducteur, évoluant en ectoderme). En retour, le mésoderme détermine les cellules à vitellus à former l'épithélium endodermique. Après quoi toutes les autres inductions s'enchaînent automatiquement, à commencer par celle de la plaque neurale lorsque le mésoderme vient se placer sous l'ectoderme. Toutes les parties de l'œuf renferment le même cytoplasme fondamental, que la transcription de la totalité du génome, au début de la méiose, a rendu totipotent [6]. C'est l'accumulation des plaquettes vitellines au pôle vitellin qui confère ses propriétés particulières à la moitié endodermique et donc programme, sans utilisation d'un code moléculaire quelconque, toutes les interactions cellulaires du développement $[7,8]$.

Parce qu'elles communiquent incessamment entre elles, les cellules se transforment et se diversifient. C'est avec des propriétés fugaces, le plus souvent banales, qu'elles créent des traits d'organisation définitifs. Les changements dans l'adhésivité des membranes plasmiques contraignent les cellules à se déplacer ou à s'agencer d'une autre manière. C'est ainsi que les ébauches des organes s'individualisent et prennent leur place définitive. Il suffit que les propriétés des membranes soient temporairement altérées pour qu'un organe n'apparaisse pas ou prenne une configuration atypique. $\mathrm{Si}$ le milieu redevient normal, la différenciation ayant progressé entre temps, les cellules utilisent maintenant à d'autres fins l'adhésivité de leurs membranes : elles ne prendront jamais leur emplacement normal. Les différences régionales dans les rythmes des divisions sont largement impliquées dans le modelage de l'organisme. Le cerveau est intéressant à cet égard parce que, ayant acquis par sa croissance une architecture compliquée, il la conserve tandis que les neurones deviennent incapables de se diviser. La cytolyse frappe des groupes entiers de cellules dont la disparition laisse des traits d'organisation indélébiles, par exemple la séparation des doigts. Ainsi, les caractères d'organisation ne reflètent pas que des activités moléculaires contemporaines contrôlées par la transcription de certains gènes. Ils sont pour la plupart des séquelles d'activités cellulaires banales et révolues [7].

Actuellement, les biologistes moléculaires progressent rapidement dans l'étude des gènes mutés connus pour leurs effets tératogènes et prédisent pour un avenir proche la compréhension du programme génétique [9]. Cependant, de leur côté, des embryologistes ont étudié les répercussions de diverses mutations sur la structuration de divers organes. Pour chaque mutation, l'origine de la malformation n'est autre chose qu'un dysfonctionnement métabolique affectant une propriété temporaire de cellules au moment où celles-ci créent un nouveau trait d'organisation. La falsification de ce trait entraîne la falsification d'autres traits mis en place ultérieurement, aussi bien dans la descendance de ces cellules que, par le jeu des interactions cellulaires, dans des populations voisines dont le métabolisme n'a pas été affecté. Les effets d'une mutation peuvent être neutralisés en redonnant un cours normal aux activités cellulaires, par un traitement approprié, administré à temps. La correction de l'anomalie structurale naissante par une intervention chirurgicale peut être aussi envisagée. Chez les oiseaux, l'anatomie de la patte est bien différente de celle de leurs ancêtres reptiliens. Il suffit de placer un écran entre les territoires présomptifs du tibia et du péroné pour que ces deux os, et la musculature qui s'y insère, se développent avec des caractères reptiliens [IO].

Les cellules sont marquées par leur appartenance à un organe : après isolement, elles en conservent au moins certaines propriétés spécifiques. Cette mémoire n'est pas dans l'ADN : chez certaines espèces, un noyau provenant de cellules différenciées d'un embryon déjà âgé est totipotent comme celui de l'œuf. La mémoire d'une cellule se présente comme des traces des activités cytoplasmiques successivement entretenues par son ascendance, en réponse aux transformations de son voisinage immédiat, résultant de la progression de la différenciation et des migrations cellulaires. Toute cellule hérite donc d'une "personnalité " particulière, déterminée par les différentes positions que son ascendance a occupées depuis le début du développement. Cette évidence est toutefois brouillée par le fait que, même chez l'adulte, les cellules doivent s'entraider pour conserver intactes les personnalités dont elles ont respectivement hérité et, du même coup, pour maintenir certains traits d'organisation formés au cours du développement.

Par cet entretien d'une "mémoire collective " (et, incidemment, par de nombreuses autres caractéristiques), le développement montre une curieuse identité de principes avec les progrès d'une civilisation. Dans une société humaine, l'individu joue un rôle qui est à la fois influencé par ce que ses progéniteurs lui ont légué et par la communication avec ses contemporains. Divers aspects de sa physiologie rendent possible et conditionnent la vie sociale, mais les principes de la vie sociale ne sont pas à rechercher dans la physiologie. Nul n'a en lui un exemplaire d'un programme de gouvernement de la société, encore moins le plan de l'histoire passée et future de la civilisation. Il en va de même pour la cellule. L'ADN qu'elle contient ne possède pas le programme du développement de l'individu auquel elle appartient : son rôle est limité, encore que partiellement, au contrôle de la synthèse des polypeptides. Toutefois, en fournissant aux cellules, notamment à l'œuf, des protéines particulières, l'ADN donne sa note originale à leur œuvre collective : la construction de l'organisme.

Il n'est pas inutile de souligner, pour terminer, que la remise en cause de la notion de programme 


\section{REFERENCES}

I. Lewin R., Why is development so illogical ? Science 1984; 224: 1327-9.

2. Ho M W, Saunders $P$ T. The epigenetic approach to the evolution of organisms - with notes on its relevance to social and cultural evolution. In: Plotkin H C, ed. Learning Development and Culture. New York: John Wiley \& Sons, 1982: 343-61.

3. Zemek K, Mlikovsky J, Socha R. Multilevel systems of heredity and its ontogenetic and phylogenetic consequences. In: Mlikovsky J, Novak V J A, cds. Evolution and Morphogenesis. Prague: Academia, 1985: 75-87.

4. Scherrer K. Cascade regulation. A model of integrative control of gene expression in eukaryotic cells and organisms. In: Kolodny G M, ed. Eukaryotic Gene Regulation. CRC Inc, 1980: 58-129.

5. Horder T J. Embryological bases of evolution In: Goodwin B C, Holder N, Wylie CG, eds Development and Evolution. Cambridge: Cambridge University Press, 1983: 31 6-52.

6. Monk M. A stem-line model for cellular and chromosomal differentiation in carly mouse development. Differentiation $1981 ; 19: 71-6$.

7. Chandebois R, Faber J. Automation in animal development. A new theory derived from the concept of cell sociology. Monogr Dev Biol 1983 ; I 6 .

8. Nieuwkoop P D, Johnen A G, Albers B. The epigenetic nature òf early chordate development. Inductive interaction and competence. In: Deve lopmental and Cell Biology Monographs, vol. I6. Cambridge: Cambridge University Press, 1985.

9. Jarry B, Grau Y. Les gènes programmeurs du développement. médecine/sciences 1985; I : 24854.

10. Müller G. Experimental reestablishment of ancestral patterns in the chick limb. In: Mlikovsky J, Novak V J A, cds. Evolution and Morphogenesis. Prague: Academia, 1985: 439-46.

I I. Stent G S. Genetic approach to developmental neurobiology. Trends Neurosci 1980; 3: 49-5I. 12. Brenner S. Genes and development. In: Lloyd C W, Ress D A, eds. Cellular Controls in Differentiation. New York: Academic Press, 1981 .

13. Changeux J P. L'homme neuronal. Paris: Fayard, 1983.

14. Palmiter R D, Brinster R L. Transgenic micc. Cell 1985; 41: $343-5$

15. Stent G S. Thinking in one dimension: the impact of molecular biology on development. Cell 1985; 40: 1-2.

16. Wilkins A S. Genetic Analysis of Animal Development. New York: Wiley and Sons, 1986.

17. Marcey D, Nusslein-Volhard C. Embryology goes fishing. Nature 1986; 321 : 380-1.

18. North G. How to make a fruitfly. Nature 1984; 3 I I: 2 1 4-6.

19. Woodland $\mathrm{H}$, Jones E. Unscrambling egg structure. Nature 1986; 319: 261-2.

20. Lawrence P A. Molecular development: is there a light burning in the hall. Cell $1985 ; 40$ : 221 génétique ne concerne en rien les acquis de la biologie moléculaire, notamment le code génétique et le contrôle de la synthèse des protéines. Elle ne concerne pas davantage la cytogénétique, puisqu'il importe peu, dans cette discipline, qu'un gène localisé sur un chromosome code pour la morphologie d'un organe ou pour une protéine dont la falsification se répercute sur la genèse de cet organe. La notion de programme génétique du développement n'a jamais été autre chose qu'une hypothèse devenue pour beaucoup une certitude, tant parut évidente la relation entre le code génétique et le rôle que Mendel avait attribué aux gènes.

\section{Rosine Chandebois}

Professeur à l'université de Provence, laboratoire de mor phogénétique animale, Université de Provence, place Vistor-Hugo, 13331 Marseille cedex 3

\section{Gènes et développement}

Il est devenu courant chez nombre de biologistes de se référer à un "programme génétique du développement " dont la mise au jour livrerait la clé des problèmes posés par l'embryologie. Il n'est pourtant pas sûr que les utilisateurs de cette expression aient toujours une idée claire de ce qu'ils y mettent. Mieux encore (ou pire ?) : il n'est pas sûr qu'on puisse donner un contenu logiquement cohérent à une telle notion, dont la critique a été amor- cée il y a une dizaine d'années par G. Stent [II] et reprise, entrc autres, par S. Brenner [12] 't J. P. Changeux [13], personnalitio qu'on ne peut guere soupçonncr d'être hostiles à la biologie moléculaire.

On sait que certains éléments du vocabulaire de l'informatique ont fait leur apparition dans la biologic au cours des années 50-60. Il se trouve que la métaphore du " programme " fonctionne particulièrement bien lorsqu'on considère la traduction d'un gène en la protéine dont il gouverne la structure. En effet, un programme informatique est constitué d'une série linéaire d'instructions. Introduit dans une machine adéquate (l'ordinateur) dont le fonctionnement est totalement indépendant du programme en question, chacune des instructions de celui-ci donne lieu à une série d'opérations dont l'ensemble finit par produire un certain résultat : solution d'une équation, classement des éléments d'un ensemble, impression d'un texte, etc. A noter, encore une fois, que le programme, la machine et le résultat constituent ici trois entités bien distinctes manipulables séparément. De la même manière, on peut dire qu'un gène est fait d'une série linéaire d'instructions (les codons). Introduit dans une machine convenable (une cellule ou, plus simplement, l'ensemble des fractions subcellulaires de la machinerie de transcription-traduction), le gène donne lieu à la fabrication d'une protéine. Tout comme précédemment, le gène, la machinerie de traduction et la protéine sont séparables. De plus, une même " machine" (cellule bactérienne ou eucaryote) peut traiter le programme de n'importe quel gène dont la provenance, bactérienne ou eucaryote, est indifférente.

$\mathrm{Si}$ maintenant l'on considère le développement embryonnaire d'un œuf d'une espèce $x$ quelconque, les choses sont bien différentes. Supposons que l'on dispose d'une préparation de l'ADN total de cette espèce, ADN qui est supposé contenir ou constituer le programme de développement. On voit immédiatement qu'il n'existe aucune machine susceptible de "traiter " cet ADN 
pour produire un individu de l'espèce $x$. La seule machine concevable serait l'œuf lui-même mais cette machine n'est nullement indépendante de l'ADN qu'on veut lui faire traiter. Sa constitution résulte en particulier de l'activité réglée de certaines parties de cet ADN au cours de l'ovogenèse. Bien plus, cette machine-œuf qu'il est dépourvu de sens d'envisager indépendamment de l'ADN ne resterait pas identique à elle-même au fur et à mesure du "traitement" de celui-ci. Elle entrerait dans le processus de construction de l'embryon et se confondrait avec le résultat attendu du traitement de l'ADN. Ainsi les trois éléments, programme, machine et résultat qui restent parfaitement séparés dans le cas d'une opération informatique comme dans celui de la lecture d'un gène sont ici inextricablement associés dans une même unité qui est l'embryon lui-même.

On dira que l'expérience qui consisterait à injecter dans l'œuf un génome entier est de toute façon parfaitement irréaliste mais qu'en revanche l'injection de gènes particuliers, assortis de séquences adjacentes en $5^{\prime}$ ou en $3^{\prime}$, de longueur variable, est maintenant réalisée couramment, par exemple dans l'œuf de souris, et permet d'observer, dans les animaux transgéniques obtenus [14], l'expression du gène dans certains lignages différenciés spécifiques. La métaphore informatique semble retrouver ici tous ses droits : un mini-programme (le gène et ses séquences adjacentes), introduit dans une machineembryon dont il est parfaitement distinct, produit comme résultat l'apparition d'une protéine dans certaines cellules bien définies. C'est peut-être l'indice que les multiples séquences de différenciation terminale qui règlent dans l'embryon l'apparition de cellules musculaires, sanguines, etc., pourraient de façon cette fois pertinente, être considérées chacune comme résultat de la lecture d'un programme élémentaire. L'embryogenèse serait alors le produit du traitement, non pas d'un programme, mais d'un programme de programmes, d'un programme d'ordre 2 en quelque sorte et plus probablement, vu la complexité du phénomène d'ordre $n$ supérieur à 2 . Il serait vain pourtant de se dissimuler que cette manière de voir ne supprime nullement la difficulté fondamentale, à savoir qu'on ne peut parler de l'ADN programme comme d'une entité extérieure à la machine qui le traite ou à l'embryon dont il est censé diriger la construction. A tout moment de l'embryogenèse, les diverses parties du génome sont dans les divers lignages cellulaires, dans des états (de condensation, d'accessibilité, d'interaction avec des protéines spécifiques, etc.) qui, à la fois, résultent des phases antérieures et déterminent les phases ultérieures de l'embryogenèse, ce qui amène $G$. Stent à parler du caractère historique plutôt que programmatique de celle-ci [15].

Au vrai, il est sans doute prématuré de prétendre dégager une théorie générale du développement à partir de principes qui seraient valables pour toute embryogenèse quelle qu'elle soit. L'embryon étant reconnu comme une structure unitaire où le degré d'intégration des parties atteint une complexité que l'on ne fait que soupçonner, le mieux qu'on puisse proposer est sans doute l'étude de séquences d'interaction très élémentaires, en espérant que, de la confrontation des situations particulières, des vérités un peu générales émergeront. Il serait probablement erroné de penser qu'un unique mode d'attaque expérimentale, celui de la biologie moléculaire par exemple, puisse être fructueux dans ce genre de recherches. L'analyse clonale du développement, réalisée chez le nématode par l'observation microscopique directe [I6], chez la drosophile par des techniques génétiques [I6] et qui est en voie de renouvellement chez les vertébrés [I7], apporte à elle seule nombre de renseignements précieux concernant les phénomènes de détermination cellulaire. C'est un exemple parmi beaucoup d'autres possibles. Mais il paraît aussi assuré que, des diverses parties dont l'embryon se compose, les gènes présents dans ses cellules et leur état d'activité importent beaucoup à la marche de l'embryogenèse, et pas seulement parce qu'ils produiraient quelques métabolites triviaux. Il existe certes une foule de mutations embryonnaires létales "inintéressantes" qui ne font que supprimer la production d'un métabolite et semblent pourtant avoir des effets morphogénétiques spécifiques en inhibant plus particulièrement la croissance cellulaire au niveau de certains organes (voir par exemple la mutation rudimentary chez la drosophile [I6]. Mais de tels cas ne devraient pas occulter l'immense intérêt qui s'attache à l'étude de mutants de la même drosophile où une antenne se change en patte parfaitement constituée, où la segmentation de l'embryon s'opère de façon anormale mais très précisément réglée, avec disparition d'un segment sur deux, où la mise en place de l'axe antéro-postérieur ou dorso-ventral de l'embryon précoce est profondément perturbée [9, I8, I9]. En fait, ce à quoi on assiste actuellement dans le cas de la drosophile, c'est à l'élucidation des circuits majeurs de régulation génétique qui conditionnent la polarité de l'œuf, la segmentation de l'embryon, puis l'identité des segments. Il est trop tôt pour dire qu'on atteindra ainsi le tout ou simplement l'essentiel de l'embryogenèse précoce de cette espèce. Il est acquis en revanche que, grâce aux techniques de la génétique moléculaire, des perspectives entièrement nouvelles sont en train d'être dégagées dont les prolongements dans l'étude des autres groupes, mammifères et homme compris, commencent seulement à être explorés [20].

\section{Hubert Condamine \\ Institut Pasteur, 28, rue du Docteur-Roux, 75724 Paris cedex 15}

